\title{
Protée
}

\section{Du mythe au concept}

\section{Barbarie et historicité dans les essais de Fatima Mernissi}

\section{Mounia Benalil}

Volume 38, numéro 3, hiver 2010

Les concepts aux frontières du savoir contemporain

URI : https://id.erudit.org/iderudit/045613ar

DOI : https://doi.org/10.7202/045613ar

Aller au sommaire du numéro

\section{Éditeur(s)}

Département des arts et lettres - Université du Québec à Chicoutimi

ISSN

0300-3523 (imprimé)

1708-2307 (numérique)

Découvrir la revue

Citer cet article

Benalil, M. (2010). Du mythe au concept : barbarie et historicité dans les essais de Fatima Mernissi. Protée, 38(3), 19-27. https://doi.org/10.7202/045613ar

\section{Résumé de l'article}

Cet article s'inscrit dans le cadre des récentes entreprises intellectuelles qui réfléchissent sur les nouveaux rapports entre l'Orient et l'Occident en examinant le vocabulaire de la mondialisation. Les essais de la sociologue marocaine Fatima Mernissi dessinent en creux les enjeux de ces rapports en posant le concept de barbarie dans le contexte de ce qu'on appelle aujourd'hui les « cultures de l'échange ». Le questionnement de la barbarie est lié à l'interrogation de la transmission du savoir par les experts masculins de la littérature religieuse islamique. L'historicité fait surgir le problème de la radicalité moderne des idéologies. Pour contrer les crispations et les dérapages qui découlent de la lecture univoque (la lecture intégriste), Mernissi propose une féminisation humaniste du savoir sur l'islam selon des procédés herméneutiques qui déconstruisent le savoir hérité. Si la barbarie est dans la transmission, la civilisation réside dans la démocratisation de la lecture du savoir.
Ce document est protégé par la loi sur le droit d'auteur. L'utilisation des services d’Érudit (y compris la reproduction) est assujettie à sa politique d'utilisation que vous pouvez consulter en ligne.

https://apropos.erudit.org/fr/usagers/politique-dutilisation/ 


\section{DU MYTHE AU CONCEPT \\ BARBARIE ET HISTORICITÉ DANS LES ESSAIS DE FATIMA MERNISSI}

MOUNIA BENALIL

Depuis quelques années, la montée de l'islamisme et d'autres formes du ressentiment rend de plus en plus difficile la compréhension de l'Islam en tant que religion et culture. L'opacité des rapports entre l'Occident et l'Orient depuis les années 1990, ravivée par les événements de l'actualité récente, a imposé et impose, pour les intellectuels des pays musulmans, le devoir de représenter autrement le savoir sur l'islam et de "dénoncer l'amalgame que les pays non musulmans [font] entre l'islamisme terroriste et l'Islam comme religion et culture» (Taboada-Leonetti, 2004: 7). Certains écrivains tels que Yasmina Khadra ont choisi de le faire à travers la voie romanesque pour dénoncer le théâtre de la violence qui agite leur pays natal ou d'autres régions du Moyen-Orient; d'autres intellectuels tels que Fatima Mernissi ont plutôt opté pour une écriture essayistique où se mêlent l'analyse sociologique et l'enquête historique pour dresser un diagnostic de "la maladie qui habite l'islam", selon l'expression de Abdelwahabb Meddeb (2002), et proposer des remèdes.

Dans cet article, je souhaite montrer comment l'écriture féminine au Maghreb, et plus particulièrement dans les essais de la sociologue marocaine Fatima Mernissi, a su, à travers un grand programme révisionniste, aller à la source de cette «maladie» par laquelle se propagent un nombre d'idées fallacieuses sur l'Islam. «L'Islam comme composante de l'identité culturelle et de la personnalité historique maghrébine" (Bekri, 1988: 42) traverse sous différents aspects la production culturelle du Maghreb. Les essais de Mernissi dessinent en creux les enjeux des rapports Orient-Occident en problématisant le concept de barbarie dans le contexte de ce qui est communément appelé les "cultures de l'échange». Dans la mondialisation actuelle, ces cultures spatialisent la modernité de ce concept dans sa fonction opératoire consistant à signifier les enjeux de notre époque ${ }^{1}$.

Il est bien sûr défendable d'avancer que, à travers la féminisation du savoir historique masculin sur l'islam proposée par Mernissi, c'est d'une nouvelle décolonisation de la révolution féministe qu'il s'agit ici, une décolonisation qui vise aussi bien à restituer aux femmes une liberté du corps et de la voix qu'à défier le savoir des experts masculins de la littérature religieuse islamique. Or, au-delà du désenchantement qui habite ces tentatives de décolonisation, il y a ce que Hegel a appelé le «saut qualitatif» sur le plan de la dialectique de la pensée, un saut que nous situons ici à la lumière de cette reconstitution féminine du savoir sur l'islam et qui 
s'accompagne de ce que Marc Angenot, théoricien du discours social, appelle, dans un autre contexte, la «rupture cognitive dans la pensée politique de gauche, [qui] intervient entre tout ce qui va, en dépit de glissements, maquillages et bricolages» (2001:85).

Ces dispositifs bricolés et branlants, poursuit Angenot, cherchent encore et toujours à réenchanter un monde opaque et intolérable sans plus avoir cependant à offrir une Réponse absolue aux fameuses questions: «D'où venons-nous? Qui sommes-nous? Où allons-nous?» (Ibid.: 63)

L'interrogation, la réorganisation, la reconstitution et l'interprétation de ce savoir sont certes des procédés de subversion, par la parole féminine, du joug de l'Histoire, de la symbolique du Patriarche et des nouvelles affres de l'extrémisme religieux. Or, pour contrer les crispations meurtrières du «désir pur» d'identité et des malaises qui découlent de l'incompréhension et des dérapages du savoir transmis sur l'islam, il ne suffit pas de contre-représenter afin d'ouvrir le dialogue; il faut proposer un programme «historiquement crédible [...] [et] dans une logique du Sens de l'histoire" (ibid.: 89) telle que nous la vivons.

Lorsqu'on examine les essais de Fatima Mernissi, notamment Sexe, Idéologie et Islam (1983), Le Harem politique (1987), Sultanes oubliées (1990), La Peurmodernité (1992), Le Harem et l'Occident (2001) et Rêves de femme. Une enfance au harem (1996) - qui est non pas un essai, mais un récit autobiographique ponctué de réflexions à valeur sociologique -, on remarque que ceux-ci soulèvent avec une particulière acuité la question de l'historicité qui fait surgir le problème de la radicalité moderne des idéologies et la nécessité du révisionnisme du savoir transmis sur l'islam par la tradition exégétique. Ce réformisme à valeur historique et culturelle s'inscrit dans la mouvance de la praxis postcoloniale en ce qu'il informe et oriente son actualité politique en tant que méthode critique qui s'intéresse, comme l'a souligné Jean-Marc Moura dans Littératures francophones et théorie postcoloniale, «aux formes de la marginalité, de l'ambiguité, aux stratégies de refus du binaire, à toutes les formes de pastiche, de parodie et de redoublements" (1999: 149-150). La démarche de Mernissi va contre la constitution monolithique de ce savoir sur l'Islam en tant que Grand Récit et privilégie une lecture en fragments à la recherche des exceptions qui créent et permettent des moments ou des brèches d'ouverture sémantique et de possibles relativisations dans l'entreprise interprétative du Coran et de la sunna (recueil des actes et des paroles du Prophète Mohammed), une entreprise jusqu'alors confiée aux experts masculins de la tradition islamique. On évoque la notion de Grand Récit dans le sens pluriel que lui donne Angenot, c'est-à-dire des

[...] systèmes idéologiques [...] qui se sont chargés de procurer aux modernes une explication historique totale balayant les horizons $d u$ passé, $d u$ présent et de l'avenir [...] [et] ont enserré l'obscurité du présent entre une explication globale du passé et une prédiction démontrée pour l'avenir. (2001: 61-62)

Je m'attacherai ici à montrer les méthodes et les objectifs des lectures du savoir islamique que propose Mernissi et leur pertinence dans le canevas des débats actuels sur l'islamisme. Car si la «barbarie» est à lire dans la transmission, la "civilisation" est à comprendre dans la démocratisation (féminine) de la lecture et de l'interprétation de ce savoir. C'est aussi dans ce sens que la sémiotique, posée dans les termes de Roland Barthes comme une critique sociopolitique engagée ou une "sémioclastie " ${ }^{2}$, s'avère utile dans l'historicisation des processus de signification et la dénonciation des (discours) métaphysiques responsables du dévidement du sens dynamique des choses.

La démarche révisionniste de Mernissi constitue dans sa structure profonde une réponse à deux ordres de discours: islamique d'une part et occidental d'autre part sur le rôle de la femme en Islam. Ce qui est mis au premier plan, c'est un retour à l'Histoire pour comprendre certains hadiths (recueils compilés des actes et des propos du Prophète Mohammed qui forment la sunna et constituent la deuxième source de la charia après le Coran) et traditions patriarcales dans leurs dimensions scénographiques, c'est-à-dire leur rôle énonciatif dans l'espace-temps de leur apparition face à l'éthique coranique elle-même. On comprend dès lors pourquoi la question du retour à l'Histoire est sous-jacente à celle des généalogies, c'est-à-dire des sources et des origines dans la construction, la 
compilation et l'interprétation imparfaite des récits de la tradition (que sont les hadiths) comparativement à la perfection achevée du Livre (le Coran).

La méthode historique permet de comprendre non seulement au sein de quel univers de sens et de référents le Coran [et par extension les Hadiths] [sont] advenu[s], mais aussi comment il[s] [sont] advenu[s]. (Benzine, 2004: 69)

Deux lignes thématiques traversent les essais de Mernissi et structurent la méthodologie de sa critique argumentative: une thématique vouée à l'analyse du portrait de la femme musulmane du point de vue de l'islam lui-même et une thématique orientée vers l'examen de ce portrait du point de vue de l'Occident. Sexe, Idéologie et Islam, Le Harem politique et La Peurmodernité sont des études consacrées au premier volet de cette analyse, alors que Sultanes oubliées, Le Harem et l'Occident et Rêves de femmes sont des écrits consacrés au deuxième volet de cette analyse. Cette division n'est pas tranchée, car les deux types d'écrits se prêtent à une lecture intertextuelle.

Sexe, Idéologie et Islam s'attaque au Code du statut personnel marocain avec une analyse pointue de «l'organisation» du contrôle de la sexualité féminine en Islam contrairement à la période préislamique, «la Jahiliya [où] promiscuité et laxisme étaient les caractéristiques d'une sexualité alors non contrôlée» (Mernissi, 1983: 31). Mernissi appuie ses propos sur les interprétations de l'imam Ghazali qui conçoit le pouvoir de la sexualité féminine comme destructeur. Elle confronte les positions de Freud avec les interprétations de l'imam Ghazali et dégage

[...] l'ironie dans le fait que les théories musulmane et européenne en arrivent à la même conclusion - la femme est une force destructrice vis-à-vis de l'ordre social, soit parce qu'elle est active, selon Imam Ghazali, soit parce qu'elle ne l'est pas, selon Freud. (Ibid.: 27-28)

Mernissi rattache son enquête à l'analyse des fondements économiques de l'anomie sexuelle dans le Maroc contemporain en montrant comment «la codification de la Sharia dans le Code moderne de la famille considère que les hommes et les femmes sont antagonistes et condamne le couple au conflit» (1983: 194). Le Harem politique apporte lui aussi une argumentation complexe autour du constat d'une réalité défavorable à la discussion du statut de la femme en Islam. Rédigé bien avant la réforme de la Moudawana (code de la famille) par Mohammed VI en 2003, Le Harem politique enquête sur un hadith misogyne attribué au Prophète et rapporté par l'imam Bokhari: «Ne connaîtra jamais la prospérité le peuple qui confie ses affaires à une femme" (dans Mernissi, 1987: 7). En rattachant la prévalence de ce hadith à la culture marocaine, Mernissi explique les circonstances et les raisons liées à sa prononciation et discute son bien-fondé. Ensuite, elle oppose la volonté philogyne du Prophète à la misogynie du calife Omar Al-Khattab pour «lier dans un même procès invisibilisation des femmes et instauration du pouvoir khalifal» (Tahon, 1996: 69). «Toute tentative de réflexion sur le problème du statut politique [de la femme en Islam] est dévorée par le débat sur ce Hadith, omniprésent et omniscient» (Mernissi, 1987: 11).

Il est crucial de souligner que Mernissi, dans son enquête historique, s'engage sur la voie de l'islam traditionnel pour montrer son ouverture à l'ijtihad (ou effort d'interprétation). Dans son ouvrage intitulé Géo-histoire de l'Islam, Pascal Buresi explique que:

[le courant traditionaliste] est ouvert à la critique moderne en ce qui concerne les hadith-s apocryphes, mais il ne rejette pas un hadîth sous prétexte qu'il n'est pas conforme à la conception que les modernes ont de la causalité historique, il se fonde sur les méthodes critiques $d u$ hadîth telles qu'elles ont été cultivées au cours des siècles. L'Islam traditionnel est certes le défenseur intégral de la Loi divine (sharîa), telle qu'elle a été comprise et interprétée au cours des siècles, mais il accepte en même temps l'effort d'interprétation (ijtihâd), en accord avec les principes juridiques traditionnels tels que qiyâs (principe d'analogie), ijmâ (consensus) et istislâh (recherche de la meilleure solution pour la communauté). (2005: 285)

En s'engageant dans la science du hadith pour discuter la question des droits politiques de la femme en Islam à partir de hadiths misogynes, Mernissi fait remarquer le nombre frauduleux des hadiths et constate que l'«une des raisons de l'inflation de faux Hadiths fabriqués [...] est que le monde musulman fut, dès la mort du Prophète, déchiré par les dissensions» (1987: 49). L'enquête de Mernissi revendique le droit qu'a 
chaque individu de juger par lui-même la véracité d'un hadith et, ce faisant, laisse voir que l'islam, "contrairement aux [avis des] jurisconsultes, laisse une place à la liberté de conscience" (Al-Naboulsi, 2006: 38). Cette liberté de conscience, endogène à l'Islam et au Coran, est ce qui doit être exploité pour dépasser le retard accumulé, selon l'avis de plusieurs historiens, depuis le XIVe siècle et

[...] la Reconquista espagnole qui [s'est terminée] par l'expulsion des musulmans et des juifs d'Espagne en 1492, date de la déconverte de l'Amérique, c'est-à-dire de l'ouverture de la route atlantique pour l'expansion de l'Europe. (Arkoun, 2005:29)

Il est plausible d'avancer que Sexe, Idéologie et Islam, Le Harem politique et La Peur-modernité dessinent en creux l'idéologie d'un parcours féministe qui se réalise dans la zone d'une confrontation idéologique entre deux pôles de référence: l'islamisme, d'une part, et la politique de l'État marocain, d'autre part. Le contrepoint est à la base de la démarche révisionniste de Mernissi. Et ce qui émane du contrepoint, c'est le refus total de ce que Caroline Fourest appelle «la tentation obscurantiste» de l'intégrisme (2005). L'éthique de l'ijtihad argumentatif, tel que Mernissi le conçoit et le pratique, doit marquer et maintenir l'opposition de la critique et amener le savoir à servir non pas une idéologie à tendance essentialiste, mais la communauté humaine, et, en l'occurrence, celle des femmes musulmanes. "Il faut reconnaître où la lettre - le Coran et la tradition - prédispose à une lecture intégriste", écrit Meddeb.

Il faut retrouver la tradition exégétique et théologique pour déceler où cette lettre offre des voies ou des encouragements à ceux qui ne retiennent de son sens que ce qui appelle à la guerre. (2002: 9)

Les courants de contestation musulmans, confusément appelés «islamisme», «fondamentalisme», «intégrisme islamiste ou musulman» ou «islam politique», semblent s'opposer à ces formes de renégociation, car ils prônent, malgré leurs divergences, un «retour à l'application [parfois aveugle] d'une certaine norme définie comme seule véritablement musulmane» (Buresi, 2005:
281) et dans laquelle la place et le rôle de la femme musulmane restent majoritairement subalternes:

[...] la femme, avec son initiative, son autodétermination, est une composante symboliquement chargée de l'ancienne et de la nouvelle Jahiliya, celle qui s'ouvre avec l'ère moderne, explique la sociologue. (Mernissi, 1983: 88)

Mernissi suit la trajectoire de l'islam politique ${ }^{3}$ qui, dans sa version non combative, est un islam d'opposition dont le militantisme se veut démocratique. Elle s'en distingue nettement, toutefois, en ceci que son propre retour au Coran et à la Tradition, pour engager politiquement et socialement le débat sur la femme et la réforme de son statut, produit des résultats différents, et ce, du moment où elle ne milite pas pour une (ré) islamisation de la société ou de la modernité face à l'occidentalisation et la mondialisation des valeurs et de la culture, ou encore face à «l'hypermodernisation du monde», pour emprunter l'expression de Gilles Lipovetsky (2004: 14). Le devenir de l'islam est au cœur du programme révisionniste de la sociologue, un devenir étroitement lié au statut de la femme qui «est le point nodal des rapports de l'Islam à la modernité et non pas seulement un thème périphérique relevant de débats féministes" (Taboada-Leonetti, 2004: 8). C'est ainsi que le salafisme (ou le retour aux sources) est à comprendre dans ses deux versions: car s'il signifie, pour certains officiels religieux, «une lecture conservatrice, passéiste de l'islam» (Boukhari, 2006: 43), il représente, pour les chercheurs progressistes en herméneutique coranique tels que Gamal Al-Banna (en Égypte) ou Mohamed Talbi (en Tunisie), une «lecture vectorielle du texte coranique mettant en relief les objectifs suprêmes de la révélation" (Benzine, 2004: 69). Gamal Al-Banna considère la charia ${ }^{4}$ comme «une base de travail [dont] il faut garder les lois compatibles avec notre époque et changer [...] les lois qui ne [le] sont pas» (Boukhari, 2006: 43). Dans le sillage de ces réflexions, on peut se demander si la modernité doit «s'accomplir par une sécularisation des institutions et des consciences ou par une réforme de la pensée religieuse” (Babès, 2004: 235). Ce questionnement soulève le débat conflictuel entre islam et démocratie, auquel Mernissi consacre son 
essai La Peur-modernité pour tenter une réponse à la question des perspectives de réformes en Islam au regard de la laïcité et qui la rattache à des réflexions similaires menées par des chercheurs tels que Abdou Filali-Ansary (2003), Abderrahim Lamchichi (2001; 2006) et Ghaleb Bencheikh (2005).

[Si] en Occident, les normes de gouvernement sont dérivées de la Magna Carta britannique, de la Révolution française ou des principes de la Déclaration américaine d'indépendance, en islam elles s'appuient sur le Coran et sur les faits et dires (hadith) du Prophète. (Fuller, 1999: 16)

Dans un même ordre d'idées, les penseurs modernes de l'Islam travaillent «à extraire les principes démocratiques du concept musulman de choura (consultation) - l'idée que le gouvernement doit refléter les désirs du peuple» (ibid.). En effet, l'islam peut répondre aux interpellations de la modernité que sont la laïcité, la démocratie et les droits de l'Homme s'il accepte de chercher, sinon de développer la laïcité à partir de son héritage propre. Si le texte coranique insiste sur une égalité fondamentale entre l'homme et la femme, il insiste aussi sur la liberté de conscience qui est le principe de base de tout raisonnement laïc. Plusieurs exemples tirés de l'analyse de Mernissi montrent comment les califes, par exemple, ont séparé le religieux du séculier ${ }^{5}$. Une séparation qui devint radicale avec Kemal Atatürk qui a aboli le Califat en 1924. Un autre exemple se rapporte aux guerres menées au temps des califes

[...] contre les apostats [les tribus qui étaient revenues sur leur conversion à l'Islam] [...] puisqu'elles ont été menées pour des impôts impayés, et non pour des raisons religieuses.

(Al-Naboulsi, 2006: 38)

Or, bien qu'elle reste dans les limites de la tradition islamique, Mernissi montre, parallèlement à ses arguments, que les valeurs laïques ne sont pas foncièrement anti-islamiques en ce qui a trait aux droits de la femme. La laïcité est non pas la négation de la religion, mais la neutralisation de son intervention dans l'espace public du Citoyen. L'auteure avance dans La Peur-modernité que:

L'humanisme laïc qui prêche la tolérance et la liberté de penser n'est pas tant une attaque contre Dieu qu'une attaque contre les fonctionnaires de l'État et l'interdiction pour eux d'utiliser les impôts et les institutions financées par les fonds publics dans le but de faire de la publicité pour la religion, quelle qu'elle soit: il est moins destiné à priver Dieu des fonds publics qu'à empêcher l'État et ses fonctionnaires d'utiliser Dieu et de l'asservir à leurs intérêts. (1992: 65)

Cela dit, Mernissi reste sceptique lorsqu'il s'agit de parler des valeurs dites modernes. Aussi les sources du féminisme musulman sont-elles, pour la sociologue, à repérer dans l'éthique coranique elle-même, puisque le Coran est «une arme politique» qui appuie l'égalité entre l'homme et la femme. Quant aux versets qui comportent des charges lourdes d'exclusion pour les femmes, et qui concernent le voile, la sexualité, l'héritage et la place de la volonté de la femme en Islam d'une manière générale, il faut dire que Mernissi ne s'y attache pas spécifiquement: elle ne se penche sur l'herméneutique coranique que pour noter la richesse de l'ambiguitté ou la contradiction interne de ces versets ${ }^{6}$, et, donc, l'ouverture continue du débat sur leur interprétation. «Le texte coranique apparaît alors comme le résultat d'une dialectique entre une parole divine et une réalité historique, humaine et culturelle» (Benzine, 2004: 69).

C'est dans ce même ordre d'idées que se situe le livre Sultanes oubliées, dans lequel Mernissi traite de figures de femmes chefs d'État en Islam pour montrer comment la gestion du politique par des reines oubliées ou ignorées de l'Histoire officielle de l'islam «est associée à l'idée de liberté, mais une liberté dans le sens de souveraineté aristocratique, et non dans le sens d'une lutte contre le despotisme» (1990: 25). La scène politique musulmane est riche de figures féminines célèbres telles que Aïcha al-Hurra, connue chez les Espagnoles sous le nom de sultane Madre de Boabdil. D'autres femmes sous les premiers califats omeyyade de Damas et abbasside de Bagdad puis mongol sont citées pour leurs qualités de stratège militaire.

Mais qu'elles soient [appelées] Khatun, malika ou sultanes, écrit Mernissi, courtisanes exerçant le pouvoir dans l'ombre à partir du harem, femmes chefs d'État officielles dont le nom est frappé par les monnaies, aucune n'a jamais porté le titre de Khalife. (Ibid.: 35) 
[Et cette] technique de gommage des détails [historiques] pertinents [sur la vie de ses reines] explique comment on se retrouve actuellement avec une mémoire musulmane uniformément misogyne, et pourquoi il faut passer au peigne fin tout découpage de l'histoire qu'on nous présente comme des vérités éternelles, et comme tradition musulmane, notamment l'association du féminin et du politique comme maléfique et porteuse de désastre. (Ibid.: 64)

Dans son étude intitulée Coran et tradition islamique dans la littérature maghrébine, Carine Bourget consacre tout un chapitre à l'œuvre d'Assia Djebar et à sa réception, et évoque particulièrement le cas de Loin de Médine qu'elle rapproche des essais de Mernissi. Bourget montre comment Djebar esquisse au fil de son roman des portraits de «femmes guerrières [...] qui se livrent à l'exégèse et n'hésitent pas à contredire l'interprétation masculine de certains versets ou Hadiths» (2002: 75) au même moment où ces guerrières contestent «la vision orientaliste de l'Islam comme un vestige du Moyen Âge" (ibid.: 81). Aussi bien l'enquête historique menée par Mernissi que la démarche romanesque de Djebar visent à décloisonner l'idéologie du harem dans sa version impériale, c'est-à-dire le harem de la débauche peint par les orientalistes où les femmes sont soumises, et non pas le harem des femmes savantes, au temps du Calife Haroun Al-Rachid et des contes arabo-persans ou encore le harem domestique des années 1950 à Fès où Mernissi a grandi. Ces deux représentations du harem, auxquelles Mernissi consacre son livre Le Harem et l'Occident, orientent la discussion sur la problématique de l'altérité, du Soi à l'Autre, et de l'«interculturation" des échanges entre l'Orient et l'Occident. L'auteure souligne dès la page inaugurale de son livre: «J'ai peur de rater le but sacré du voyage: comprendre les étrangers que je vais rencontrer sur mon chemin» (2001: 7).

En puisant ses arguments dans la tradition musulmane, Mernissi rompt, d'une certaine manière, avec le féminisme occidental qu'elle ne considère pas comme le marqueur historique du féminisme arabomusulman. Dans Rêves de femmes, elle insiste sur l'idée:

[que] la libération des femmes n'est pas une idée importée de Paris ou New York, mais bien une idée endogène à la dynamique arabe et musulmane, et qui a mûri au sein des grands centres de la pensée musulmane comme les universités al-Azhar (Égypte), Zitouna (Tunis) et Quaraouiyine (Maroc).

(1996: 236)

Dans un chapitre du même livre, intitulé «Les féministes égyptiennes visitent la terrasse», elle relate le théâtre imaginaire de ses tantes Chama et Habiba où plusieurs héroïnes de la culture arabo-musulmane sont mises en scène et nourrissent les aspirations des femmes marocaines. Or, si «la femme habitant l'imaginaire masculin oriental est "dangereuse" et "subversive" "(Kilani, 2004: 108), étant souvent représentée "comme le facteur principal de la fitna - de la division et de la dissension - au sein de la Communauté des croyants» (ibid.: 109), sinon comme "victime [...] des rituels de rééquilibrages", selon la fameuse formulation de Zakya Daoud (2000: 68), il faut dire que la femme occidentale n'échappe pas à cette instrumentalisation de la femme à des fins idéologiques. À ce titre, explique Bourget,

Mernissi porte un regard très lucide sur l'Occident et sur des formes d'oppression de la femme qui finissent par passer inaperçues. Elle établit un parallèle entre l'exploitation $d u$ corps féminin en Islam (par le voile et la réclusion) et en Occident (par l'exploitation de sa nudité à des fins commerciales). (2002: 49)

Un recadrage de la question féministe selon deux points de vue et un déplacement constant de perspectives sont donc inévitables dans toute approche de la situation de la femme en regard de l'islam, et ce, d'autant plus que la vision totalitaire de l'islam politique consolide l'idée de l'enfermement de la femme et de sa manipulation symbolique.

Dans «Le mythe aujourd'hui», Barthes a distingué entre deux types de langage: "le langage objet qui parle les choses, [...] [et] le méta-langage, qui parle des choses" (1957 : 231). Cette différenciation l'amène à poser le mythe comme un métalangage où l'Histoire se transforme en nature, la fonction idéologique du mythe étant de désinvestir toute parole sur le monde et sur autrui de sa substance réelle, politique ou historique. Chez Mernissi, la révision métahistorique 
des hadiths, des documents officiels sur le rôle politique occulté de la femme en Islam ainsi que des écrits qui forment dans leur ensemble ce qu'elle appelle le "harem des Occidentaux" (2001: 20) s'oppose à tout métalangage où l'histoire de la femme en Islam risquerait justement de se transformer en nature, sans opacité aucune. La féminisation de ce nouveau savoir est une forme de subversion ou de détrônement du savoir doxique où se reflètent les limites de la représentation historique de la femme en Islam. Rappelons avec Angenot que:

[les] croyances doxiques ont une tendance à l'inertie. [...] En ce sens, tout ce que présuppose l'individu doxique est formé de préjugés. Toutes ses notions sont des prénotions [...], ses schémas sont des préconstruits [...], ils sont des cadres fixes qui encadrent le nouveau avec des opérations mentales familières. [...] On lui oppose une raison active, non précontrainte, libre de ses démarches et libre de s'éloigner des sentiers battus (c'est ce que dit le préfixe para - dans paradoxe, à côté de).

(2008: 422-423)

Si l'Histoire produit ainsi son antithèse qui est le mythe, la réécriture de cette Histoire suppose la production d'un «type de discours qui [ferait] à l'intérieur de lui-même l'épreuve de l'altérité» (Mari, 1984: 109). Le titre du livre incontournable de Jean-François Mattéi, La Barbarie intérieure. Essai sur l'immonde moderne, résume bien cette constatation dans la mesure où Mernissi, dans son entreprise révisionniste, travaille elle aussi à dégager la «barbarie intérieure» qui ronge l'islam avec ses deux «constantes» qui reviennent d'une manière quasi stéréotypique dans les discours, à savoir la «soumission de la femme [et] [l']amalgame entre la religion et l'État» (Gozlan, 2002: 105) et «la barbarie extérieure", qui émane de l'hégémonie culturelle de l'Occident et de l'histoire du colonialisme étant donné, comme l'explique Bourget, que «la situation de la femme fut brandie comme preuve de l'infériorité des pays musulmans par l'Occident afin de justifier leur colonisation» (2002: 40). La relecture historique de l'héritage musulman, animée par le désir de provoquer des changements dans la condition de la femme au Maroc, se manifeste dans la façon qu'a Mernissi de déjouer ou de délocaliser les instances du politique ou du pouvoir (le triptyque état/patriarcat/ religion) à travers un ijtihad traversé par l'écriture du corps, de la voix, de la mémoire surtout et de la subversion des binarités limitrophes qui vont contre la fabrique polyphonique du sens.

De l'amnésie comme mémoire, du passé comme déformation des possibilités du présent. [...] Étrange destinée que celle de cette mémoire musulmane que beaucoup interpellent pour censurer et punir. (Mernissi, 1987: 245)

La démarche de Mernissi est moderniste car, en se réalisant dans la logique de l'ijtihad argumentatif et du rêve du renouveau sociopolitique du Maroc et de la condition de ses femmes, elle lance un défi d'opposition, sinon de discussion ouverte avec ce que le théoricien du dialogisme Mikhail Bakhtine désigne par le «donné et le créé dans l'énoncé verbal» (1984: 329). Le donné se situe ici sur le plan des textes de loi islamiques et de leur interprétation par les experts masculins de la science religieuse, alors que le créé réside sur le plan de la métahistoire ou de l'ijtihad métahistorique tel que pratiqué par Mernissi. La "transfiguration", selon Bakhtine, du donné en créé fait réfléchir sur la fonction transitive de l'écriture, sur les mécanismes de passage d'une discipline à l'autre, d'une esthétique à l'autre dans la quête d'une forme d'écriture capable de rendre compte du rapport du texte à l'historicité: «l'objet "islam” exige chaque fois une construction analytique en fonction du contexte et des intentions des acteurs sociaux" (Kilani, 2004: 103). Au milieu des malentendus et des mésententes entre traditionalistes et progressistes, les essais de Mernissi cherchent, à leur manière, à dissocier la bêtise du bon sens et à déconstruire le discours doxique du «fait islamique» qui fait et veut faire de l'islam l'ennemi des catégories les plus belles et les plus humaines de la pensée, à savoir la science, l'Histoire, la raison et l'amour.

La modernité, explique Angenot, peut être vue comme un apprentissage, toujours inachevé parce que se heurtant toujours à des résistances fantasmatiques nouvelles, du désenchantement. (2001: 92)

Ce qui désenchante Mernissi, c'est le ressentiment sous toutes ses formes et les discours des producteurs 
ou «idéologue[s] du ressentiment» (ibid.: 144) qui cherchent «à refétichiser la tradition, la morale, la nation, le groupe, la famille» (ibid.: 143) par voie de replis contraires au progrès et aux exigences des aspirations démocratiques.

Qu'on ait, au cours des siècles, assisté à la liquidation $d u$ croyant-qui-critique-et-qui-juge et à son remplacement par un musulman-muselé-censuré-obéissant-et-reconnaissant en plus, n'enlève rien à cette dimension fondamentale de la science religieuse, [son ancrage nécessaire dans le vécu des hommes et son ouverture primordiale au débat]. (Mernissi, 1987: 49)

Dans cette perspective, on peut dire que Fatima Mernissi est une «enfant» parmi «les enfants de Rifaa", par allusion au livre phare de Guy Sorman, Les Enfants de Rifaa, et au réformateur égyptien du XIXe siècle, Rifaa el-Tahtawi. Les successeurs de Rifaa sont ces progressistes musulmans qui, du Maroc au Bangladesh, en passant par l'Indonésie, l'Égypte, la Turquie, l'Arabie saoudite et l'Iran, militent pour sauver l'intégrité de l'islam de l'islamisme radical ou des idéologies totalitaires des gouvernements qui le pratiquent ou des «fous d'Allah» qui le propagent.

\section{NOTES}

1. Par «signifier», j'entends « rendre compte des structures de signification aptes à mettre en discours et à rationaliser sémiotiquement l'interprétation des phénomènes de notre histoire contemporaine". 2. Barthes précise dans une version rééditée de Mythologies: «pas de dénonciation sans son instrument d'analyse fine, pas de sémiologie qui finalement ne s'assume comme une sémioclastie» (1970: 7).

3. On se réfère ici à la définition donnée par Pascal Buresi dans Géo-histoire de l'Islam. «D'une certaine manière, explique l'auteur, parce qu'il est tout entier consacré au changement et à la réforme et qu'il accorde une grande importance à l'organisation de la société, l'"islam politique" est "moderne" [...]. Il est ainsi surtout un fait de société qui s'inscrit dans deux continuités : celle de la réforme, avec un accent mis sur les mœurs et sur le puritanisme, et celle de l'anti-colonialisme, qui se confond souvent avec un anti-occidentalisme, surtout après 2003 et les deux guerres du Golfe» (2005: 287-288).

4. «La totalité des commandements de Dieu, tels qu'ils sont énoncés dans le Coran et les Traditions, selon les principes analytiques des quatre écoles juridiques orthodoxes" (Thoraval, 2001 : 68).

5. Le Maroc, qui est «l'expression vivante d'un mode de gouvernement islamique, aux antipodes de la laïcité occidentale" (Gozlan, 2002: 150) en raison notamment du caractère religieux de la légitimité monarchique, est un cas intéressant de paradoxes où l'évolution laïque se fait à l'intérieur des codes et des lois qui régissent le gouvernement. En témoigne la réforme de la Moudawwana qui s'inscrit dans «le processus de transition démocratique entamé depuis l'accession au trône [de Mohammed VI] [et dont le but est d']ancrer davantage le Maroc dans la modernité" (Lamchichi, 2006: 168), et ce, malgré la «menace intégriste et la majorité conservatrice qui y voit une atteinte aux fondements islamiques de la société marocaine " (ibid.: 162). En plus, l'islam marocain n'est pas l'islam saoudien qui n'est pas l'islam turc ou l'islam algérien. Et cette seule différence suffit à rappeler le rôle du contexte et de la culture dans la pratique politique et éducative en contexte islamique.

6. Pour un repérage de ces versets, voir Jean-Paul Roux (2006) et sur l'ambiguité sémantique dans le Coran, voir Olfa Youssef (2007). 


\section{RÉFÉRENCES BIBLIOGRAPHIQUES}

AL-NABOUlsI, S. [2006]: "Pour une laïcité musulmane", Courrier international, no809, du 4 au 10 mai, 38.

ANGENOT, M. [2001]: D'où venons-nous? Où allons-nous? La décomposition de l'idée de progrès, Montréal, Éd. Trait d'union;

[2008]: Dialogues de sourds: doxa, idéologies, coupures argumentatives. Traité de rhétorique antilogique, Paris, Mille et une nuits. ARKOUN, M. [2005]: Humanisme et Islam. Combats et propositions, Paris, Vrin.

BABĖS, L. [2004] : «Féminisme, islamisme, modernité », dans I.

Taboada-Leonetti, 235-246.

BAKHTINE, M. [1984] : Esthétique de la création verbale, Paris, Gallimard. BARTHES, R. [1957]: «Le mythe aujourd'hui », Mythologies, Paris, Seuil, 191-247;

[1970]: Mythologies, Paris, Seuil.

BEKRI, T. [1988] : «Islam, tradition et modernité dans la littérature maghrébine de langue française", Notre librairie, no95, 41-46.

BenCHeIKH, G. [2005]: La Laïcité au regard du Coran, Paris, Presses de la Renaissance.

BENZINE, R. [2004]: "Le Coran entre transcendance et histoire", Le Nouvel Observateur (hors série: "Les nouveaux penseurs de l'islam »), avril-mai, 68 .

BOUKHARI, K. [2006]: «L'homme compte plus que le texte sacré», Courrier international, no 809, 4 au 10 mai, 43.

BOURGET, C. [2002]: Coran et tradition islamique dans la littérature maghrébine, Paris, Karthala.

BuRESI, P. [2005]: Géo-histoire de l'Islam, Paris, Éd. Belin.

DAOUD, Z. [2000]: «L'implication des femmes dans le politique au Maghreb", dans C. Lochon, V. Bodin et J.-P. Doumengue (dir.), Femmes et Islam. Actes du colloque «Rôle et statut des femmes dans les sociétés contemporaines de tradition musulmane" (Paris, 15-16 décembre 1999), Paris, Centre des hautes études sur l'Afrique et l'Asie moderne, 64-78

FILALI-ANSARY, A. [2003]: Réformer l'islam? Une introduction aux débats contemporains, Paris, Éd. La Découverte.

Fourest, C. [2005]: La Tentation obscurantiste, Paris, Grasset.

FULLER, G. [1999]: «De puissantes forces modernisatrices», Le Monde diplomatique, septembre, 16-17. En ligne: http://www.mondediplomatique.fr/1999/09/FULLER/12435 (page consultée le 26 octobre 2010).
Gozlan, M. [2002]: Pour comprendre l'intégrisme islamiste, Paris, Albin Michel.

KILANI, M. [2004] : «Femmes, religion et islam. De quelques constructions hégémoniques ", dans I. Taboada-Leonetti, 95-110.

LAMCHICHI, A. [2001]: Géopolitique de l'islamisme, Paris, L'Harmattan; [2006]: Femmes et Islam. L'impératif universel d'égalité, Paris,

L'Harmattan.

Lipovetsky, G. et J. Serroy (2004): La Culture-monde, Paris, Odile

Jacob.

MARI, P. [1984]: "Du roman au carnaval: le corps introuvable», Esprit, vol. 8 , no 7-8, 104-114.

MATTÉI, J.-F. [1999]: La Barbarie intérieure. Essai sur l'immonde moderne, Paris, PUF.

Meddeb, A. [2002]: La Maladie de l'islam, Paris, Seuil. Mernissi, F. [1983]: Sexe, Idéologie et Islam, Paris, Éd. Tierce; Michel; [1987]: Le Harem politique. Le Prophète et les femmes, Paris, Albin [1990]: Sultanes oubliées. Femmes chefs d'État en Islam, Paris, Albin Michel;

[1992]: La Peur-modernité. Islam et démocratie, Paris, Albin Michel; [1996]: Rêves de femmes. Une enfance au harem, Paris, Albin Michel;

[2001]: Le Harem et l'Occident, Paris, Albin Michel.

MOURA, J.-M. [1999]: Littératures francophones et théorie postcoloniale,

Paris, PUF.

RouX, J.-P. [2006]: Les Ordres d'Allah, Sur l'homme, la société, la famille, la femme et les rapports avec les autres, Paris, Desclée de Brouwer.

SORMAN, G. [2003]: Les Enfants de Rifaa. Musulmans et modernes, Paris, Fayard.

TABOADA-LEONETTI, I. (dir.) [2004]: Les Femmes et l'Islam. Entre modernité et intégrisme, Paris, L'Harmattan.

TAHON, M.-B. [1996]: "Les femmes et le religieux chez Fatima Mernissi ", dans Y. Bénayoun-Szmidt, H. Bouraoui et N. Rédouane (dir.), La traversée du français dans les signes littéraires marocains. Actes du colloque international de l'Université York (Toronto, Canada), 20-23 avril 1994, Toronto, Éd. La Source, 65-75.

THOROVAL, Y. [ (1995) 2001]: Dictionnaire de civilisation musulmane, Paris, Larousse/Bordas.

YousseF, O. [2007]: Le Coran au risque de la psychanalyse, Paris, Albin Michel. 